\title{
THY1 is a conserved marker of undifferentiated spermatogonia in the pre-pubertal bull testis
}

\author{
Suzanne C Reding, Aaron L Stepnoski, Elizabeth W Cloninger and Jon M Oatley \\ Department of Dairy and Animal Science, Center for Reproductive Biology and Health, The Pennsylvania State \\ University, University Park, Pennsylvania 16802, USA \\ Correspondence should be addressed to J M Oatley; Email: jmo15@psu.edu
}

\begin{abstract}
The undifferentiated spermatogonial population consists of stem and progenitor germ cells which function to provide the foundation for spermatogenesis. The stem cell component, termed spermatogonial stem cells (SSCs), is capable of self-renewal and differentiation. These unique attributes have made them a target for novel technologies to enhance reproductive function in males. With bulls, culture and transplantation of SSCs have the potential to enhance efficiency of cattle production and provide a novel avenue to generate transgenic animals. Isolation of SSCs is an essential component for the development of these techniques. In rodents and non-human primates, undifferentiated spermatogonia and SSCs express the surface marker THY1. The hypothesis tested in this study was that THY1 is a conserved marker of the undifferentiated spermatogonial population in bulls. Flow cytometric analyses showed that the THY1 + cell fraction comprises a rare sub-population in testes of pre-pubertal bulls. Immunocytochemical analyses of the isolated THY1 + fraction for expression of VASA showed that this cell population is comprised mostly of germ cells. Additionally, expression of the undifferentiated spermatogonial specific transcription factor promyelocytic leukemia zinc finger (PLZF, ZBTB16) protein was found to be enriched in the isolated THY1 + testis cell fraction. Lastly, xenogeneic transplantation of bull testis cells into seminiferous tubules of immunodeficient mice resulted in greater than sixfold more colonies from isolated THY1 + cells compared to the unselected total testis cell population indicating SSC enrichment. Collectively, these results demonstrate that THY1 is a marker of undifferentiated spermatogonia in testes of pre-pubertal bulls, and isolation of THY1 + cells results in their enrichment from the total testis cell population.
\end{abstract}

Reproduction (2010) 139 893-903

\section{Introduction}

Spermatogenesis is a highly specialized and productive tissue system producing millions of spermatozoa every day (Sharpe 1994). Continual production depends on proliferation of the undifferentiated spermatogonial population which consists of type $A$ single $\left(A_{s}\right)$, $A$ paired $\left(A_{p r}\right)$, and $A$ aligned $\left(A_{a l}\right)$ spermatogonia. The $A_{s}$ spermatogonia have traditionally been considered the spermatogonial stem cell (SSC) population capable of infinite self-renewal and differentiation which produces $A_{p r}$ followed by $A_{a l}$ spermatogonia giving rise to differentiating spermatogonia that are committed to eventual production of spermatozoa (Huckins 1971, Oakberg 1971). Postnatally, gonocytes (i.e. pre-spermatogonia) are the first testicular germ cells and undergo transition into undifferentiated spermatogonia during a defined period of development that varies among species. In mice, this transition occurs between 0 and 6 days post partum (dpp), but in bulls conversion of gonocytes is not complete until 6 months of age (Curtis \& Amann 1981). Thus, during a certain period of development, the germ cell population is a heterogeneous mix of gonocytes and undifferentiated spermatogonia, a population that can be described as primitive spermatogonia. Molecular markers have been used to identify and study undifferentiated spermatogonia and gonocytes. Promyelocytic leukemia zinc finger protein (PLZF) is expressed exclusively by the undifferentiated spermatogonial population in rodents, nonhuman primates, and pigs (Buaas et al. 2004, Costoya et al. 2004, Hermann et al. 2007, Luo et al. 2009). Ubiquitin carboxyl-terminal esterase L1 (UCHL1) is a general marker of spermatogonia in the bull (Herrid et al. 2007) and boar (Frankenhuis et al. 1982, Luo et al. 2006, 2009). Additionally, expression of VASA (DDX4) has been localized to several sub-types of spermatogonia in many species including bulls (Bartholomew \& Parks 2007), boars (Lee et al. 2005), primates (Hermann et al. 2007), and mice (Toyooka et al. 2000).

In livestock populations, a majority of genetic gain is made through the male germline. Thus, isolation, culture, and transplantation of SSCs are tools that could enhance the efficiency of food animal production. Maintenance of bovine SSCs in culture would allow for immortalizing the germline of bulls with desirable 
genetics. Transplanting SSCs from a donor bull into recipient males would present a novel means for efficiently expanding the availability of genetics for specific sires in commercial livestock populations. Additionally, culture and transplantation of bovine SSCs could provide a novel avenue for generation of transgenic livestock. Knowledge of the undifferentiated spermatogonial population in bulls is limited, and the development of SSC applications has been hampered due to inability to isolate these cells from the total testis cell population.

Currently, methods for efficiently culturing and transplanting SSCs are only available for rodents (Brinster \& Avarback 1994, Brinster \& Zimmermann 1994, Kubota et al. 2004) and have yet to be fully established for livestock species, though some initial progress has been made (Dobrinski et al. 2000, Izadyar et al. 2003, Oatley et al. 2002, 2004b, Aponte et al. 2008). Development of these procedures in rodents has been aided greatly by identification of surface markers expressed by undifferentiated spermatogonia, allowing for isolation of cell fractions enriched for these cells compared to the total testis cell population. For rodents and non-human primates, selection of CD90 or THY1-expressing testis cells results in enrichment of SSCs (Kubota etal. 2003, Ryu et al. 2004, Hermann et al. 2009). Selection of THY1 + cells from testes of adult mice produces an enrichment of $>30$-fold for SSCs compared to the total testis cell population (Kubota et al. 2003), and studies by Ryu et al. (2004) revealed that all SSCs in the rat testis are $\mathrm{THY} 1+$. Importantly, selection of THY1 + cells from testes of rhesus macaques also results in enrichment of SSCs compared to the unselected total testis cell population (Hermann et al. 2009). Collectively, these previous studies indicate a conserved phenotype of THY1 expression by SSCs in mammals. To date, expression of THY1 in the germline of any livestock species has not been reported. The objective of the current study was to isolate a testis cell fraction enriched for undifferentiated spermatogonia from the testes of pre-pubertal bulls. To achieve this, the hypothesis tested was that THY1 is a conserved marker of undifferentiated spermatogonia in the bull.

\section{Results}

\section{THY1 is expressed by a rare sub-population of spermatogonia in pre-pubertal bull testes}

In the testes of adult mice, SSCs are a rare germ cell subpopulation, and selection of cells expressing the surface antigen THY1 results in their enrichment (Brinster \& Zimmermann 1994, Dobrinski et al. 2000). Here, we examined expression of THY1 in testes of pre-pubertal bulls. Using flow cytometric analyses (FCA), the THY1expressing cell fraction was determined to represent $0.05 \pm 0.01 \% \quad(n=3$ replicate testis cell preparations from different bulls) of the total testis cell population in pre-pubertal bulls (Fig. 1A). Immunohistochemistry
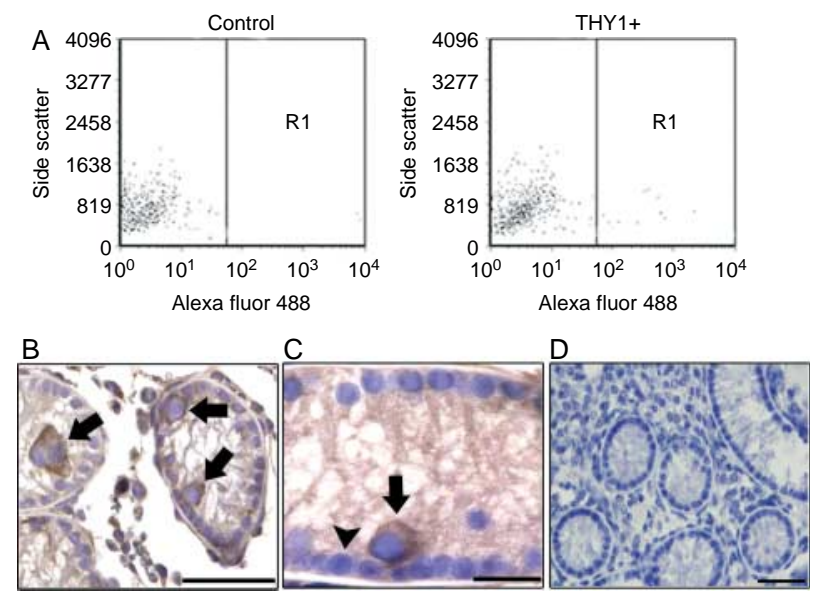

Figure 1 Examination of the THY1-expressing cell fraction in pre-pubertal bull testes. (A) Representative dot plots of flow cytometric analyses for the percentage of cells expressing THY1 in testes of pre-pubertal bulls. On average, $0.05 \pm 0.01 \%(n=3$ different cell preparations from individual bulls) of cells in pre-pubertal bull testes were THY1 + (R1 gate) when compared to unstained control cells. (B and C) Immunohistochemical examination of THY1-expressing germ cells (arrows) within cross sections of seminiferous tubules from pre-pubertal bulls. Sertoli cells (arrowhead), identified based on morphology of their distinct nucleoli, did not stain for THY1 expression. Bars are $50 \mu \mathrm{m}$. (D) Image of negative control staining in which normal mouse IgG was used as the primary antibody. Bar is $50 \mu \mathrm{m}$.

analysis of cross sections from pre-pubertal bull testes revealed that THY1 expression is localized to spermatogonia along the basement membrane of seminiferous tubules (Fig. 1B and C). To determine the germ cell content of the THY1 + cell fraction, magnetic cell sorting
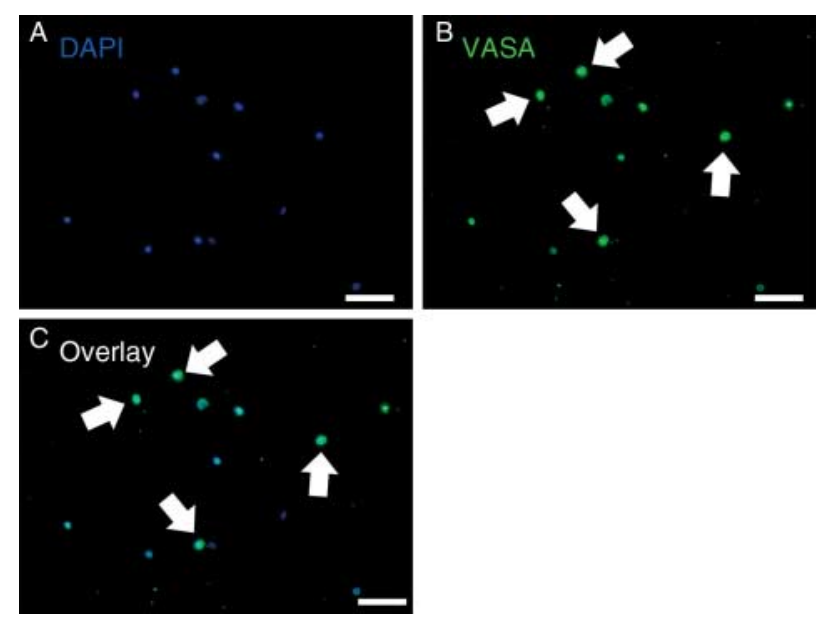

Figure 2 Examination of the germ cell content of the MACS-isolated THY1 + cell fraction from pre-pubertal bull testes. (A) DAPI staining to label nuclei of individual cells from the MACS-isolated THY1 + cell fraction. (B) Immunofluorescent staining of a single cell suspension of MACS-isolated THY1 + testis cells for expression of the germ cell-specific marker VASA. Arrows indicate VASA + cells. (C) Overlay of DAPI- and VASA-stained images. Bars are $100 \mu \mathrm{m}$. On average, $84.2 \pm 0.7 \%$ of cells were VASA + . 
methodology, MACS, was used to isolate THY1 + cells, and this population was examined for expression of the germ cell-specific marker, VASA. These analyses revealed that $84.2 \pm 0.7 \% \quad(n=2$ different cell preparations) of the MACS-isolated THY1 + cells were germ cells (Fig. 2). Collectively, these results suggest that similar to rodents and non-human primates, THY1 is expressed by a rare sub-population of spermatogonia in the testes of pre-pubertal bulls.

\section{Expression of PLZF is localized to a sub-population of spermatogonia within seminiferous tubules of pre-pubertal bulls}

Previous studies revealed that UCHL1 is a general marker of type A spermatogonia in the bull testis (Herrid et al. 2007). In rodents and non-human primates, expression of PLZF (ZBTB16) is localized specifically to undifferentiated spermatogonia including SSCs (Buaas et al. 2004, Costoya et al. 2004, Hermann et al. 2007, Luo et al. 2009); however, expression of this molecule has not been evaluated as a marker of spermatogonia in the bull. Immunofluorescent analysis of cross sections from testes of pre-pubertal bulls revealed PLZF expression by selection of spermatogonia
(Fig. 3A). Interestingly, all spermatogonia did not stain for PLZF expression, and further examination revealed the presence of $0.9 \pm 0.15$ PLZF + germ cells per seminiferous tubule (Fig. $3 \mathrm{~A}$ and $\mathrm{F} ; n=3$ cross sections from different bulls). Both nuclear and cytoplasmic expression of PLZF was observed. In comparison, expression of the general spermatogonial marker UCHL1 was observed only in the cytoplasm of spermatogonia at a frequency of $2.6 \pm 0.29$ positive cells per seminiferous tubule (Fig. $3 \mathrm{~B}$ and F; $n=3$ cross sections from different bulls). These results suggested that PLZF expression is restricted to a sub-population of spermatogonia in the pre-pubertal bull testis. To examine this possibility, co-immunofluorescent analysis was conducted for both PLZF and UCHL1. In agreement with our prediction, only a portion of spermatogonia were found to express both PLZF and UCHL1 in the prepubertal bull testis with all PLZF + cells also being UCHL1+ (Fig. 3C and D). Some seminiferous tubules contained $\mathrm{UCHL} 1+$ spermatogonia only, whereas other contained both UCHL1+ and PLZF/UCHL1+ spermatogonia (Fig. 3F). These observations demonstrate that PLZF expression is restricted to a subpopulation of spermatogonia in pre-pubertal bull testes which may be SSCs. In consideration of the above
A

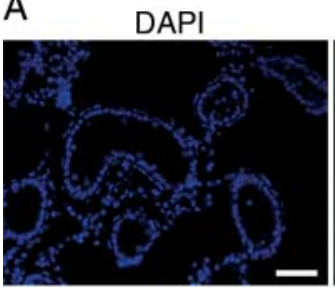

B

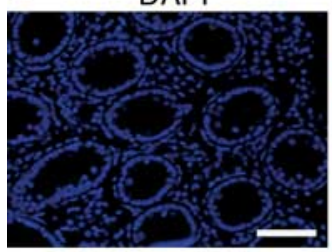

C DAPI / PLZF / UCHL1

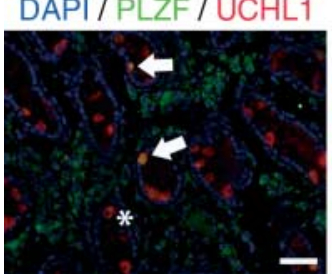

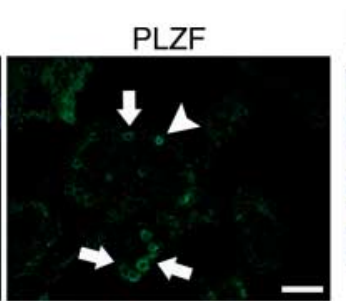

E

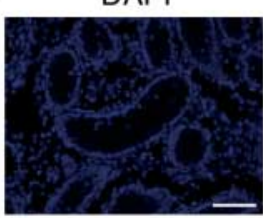

DAPI

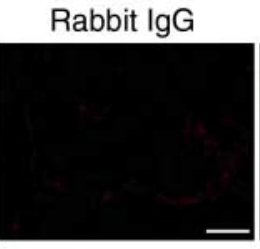

$\mathrm{UCHL} 1$

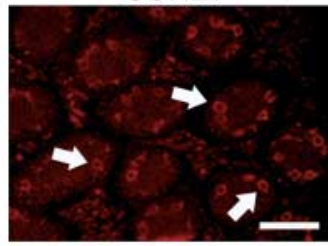

D

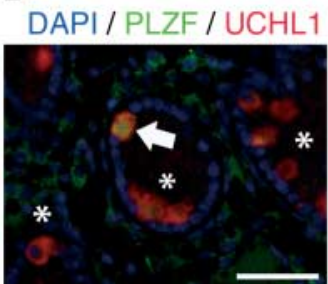

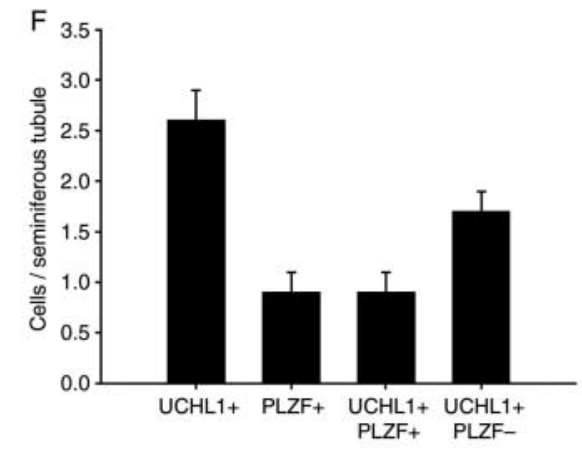

Figure 3 Identification of PLZF + spermatogonia in pre-pubertal bull testes. Immunofluorescence examination of cells expressing PLZF (A) and UCHL1 (B) within the seminiferous tubules of pre-pubertal bulls. Arrows indicate PLZF + or UCHL1 + germ cells. (C and D)

Co-immunofluorescence analyses of cells expressing PLZF (green) and UCHL1 (red) in the testes of pre-pubertal bulls. All PLZF + spermatogonia also expressed UCHL1 (yellow/orange stained, arrows), whereas some UCHL1 + cells were not PLZF+ (red stained only, asterisks). Figure D is an increased magnification of C. (E) Images of negative control staining for PLZF and UCHL1 in which normal rabbit IgG (control for UCHL1 staining) and normal goat IgG (control for PLZF) were used as the primary antibodies. Bars are $50 \mu \mathrm{m}$. (F) Number of UCHL1 +, PLZF+,

UCHL1 +/PLZF +, and UCHL1 +/PLZF - cells per seminiferous tubule. Data are mean \pm s.E.M. for three different replicate bulls. 
results, PLZF expression was used as an indicator of enrichment for undifferentiated spermatogonia and possibly SSCs in further experiments.

\section{The THY1 + cell fraction of pre-pubertal bull testes is enriched for undifferentiated spermatogonia}

Next, we examined PLZF expression in the THY1 + cell fraction isolated by MACS from pre-pubertal bull testes. Immunocytochemical analysis of single cell suspensions revealed that the isolated THY1 + cell fraction contains a significantly $(P=0.001)$ greater percentage of PLZF + cells compared to the unselected testis cell population (Fig. 4A and B). On average, $64.4 \pm 5.0 \%(n=3$ cell populations from different bulls) of cells in the THY1+ population were PLZF+, whereas only $24.7 \pm 3.5 \%$ $(n=3)$ of cells in the unselected total testis cell population expressed PLZF (Fig. 4C). Thus, MACS isolation of THY1 + cells yielded a testis cell population enriched 2.6-fold (64.4/24.7) for PLZF + spermatogonia compared to the unselected total testis cell population. Examination of gene expression with quantitative reverse transcription-PCR (qRT-PCR) analyses revealed a significant $(P=0.04)$ increase of $15.6 \pm 6.3$-fold $(n=3$ cell populations from different bulls) for ZBTB16 (PLZF) expression in the THY1 + cell fraction compared to the unselected total testis cell population (Fig. 5A). Additionally, expression of $B C L 6 B$, another molecular marker highly expressed by the SSC-enriched THY1+ cell fraction in rodents (Oatley et al. 2006, 2007), was also significantly greater $(P \leq 0.001)$ in THY $1+$ cells compared to the unselected total testis cell population (Fig. 5B). Western blot analysis revealed that PLZF protein expression was also significantly $(P=0.05)$ greater by $8.2 \pm 5.0$-fold $(n=3$ cell populations from different bulls) in the THY1 + cell fraction of pre-pubertal bulls compared to the unselected total testis cell population (Fig. 5C and D). Together, these results indicate that the THY $1+$ cell fraction from pre-pubertal bull testes is enriched for undifferentiated spermatogonia.

\section{The THY1 + cell fraction in pre-pubertal bull testes is enriched for germ cells capable of colonizing recipient testes following transplantation}

Stem cells are defined by a functional ability to colonize and reestablish homeostasis of a tissue system. The only means to unequivocally assess SSC content of an experimental cell population is by transplantation into recipient seminiferous tubules upon which SSCs colonize and reestablish spermatogenesis (Brinster \& Avarback 1994, Brinster \& Zimmermann 1994). Unfortunately, efficient and reproducible SSC transplantation between bulls is not available. However, a xenogeneic transplantation assay in which bovine germ cells are microinjected into seminiferous tubules of recipient immunodeficient mice provides an assessment of possible stem cell content based on the formation of colonies of bovine cells (Dobrinski et al. 2000, Oatley et al. 2004a, 2004b). To assay for possible SSC enrichment, MACS-isolated THY1 + cells from prepubertal bull testes and corresponding unselected total testis cell populations were labeled with a fluorescent membrane linker dye and microinjected into seminiferous tubules of immunodeficient recipient mice. The number of fluorescently labeled bovine germ cell colonies was then evaluated in dispersed seminiferous tubules 4 weeks later. Regardless of the cell suspension, fluorescent colonies of bovine germ cells were observed

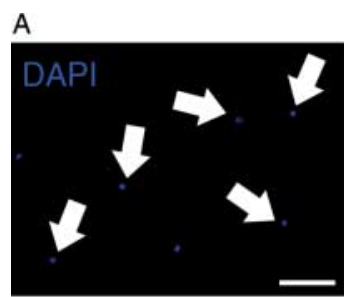

B

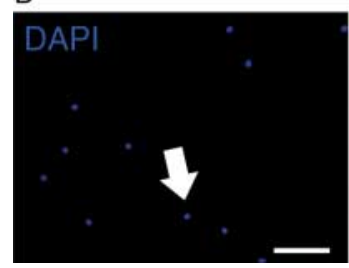

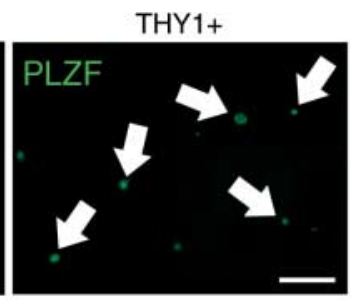

Unselected

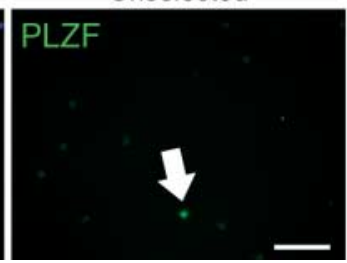

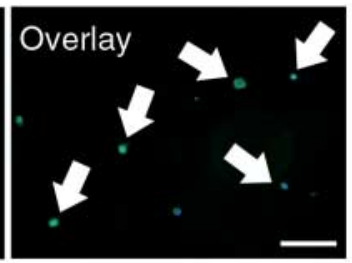

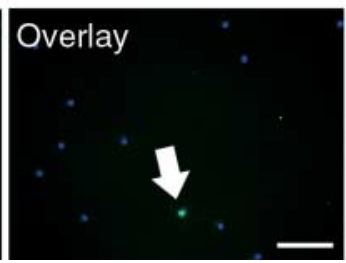

C

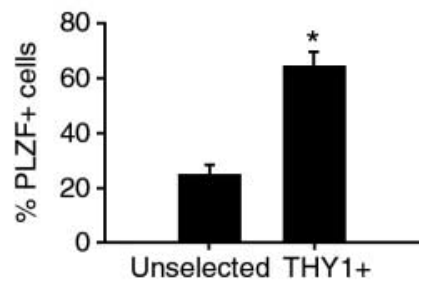

Figure 4 Examination of the MACS-isolated THY1 + cell fraction from pre-pubertal bull testes for PLZF-expressing cells. Representative images of immunocytochemical analyses for PLZF+ cells in MACS-isolated THY1 + cell fractions (A) and unselected total testis cell populations (B). Arrows indicate PLZF + cells. Bars are $100 \mu \mathrm{m}$. (C) Quantitative comparison of immunocytochemical analyses for the percent PLZF + cells in MACS-isolated THY1 + cell fractions and unselected total testis cell populations. Data are mean \pm s.E.M. for three different cell preparations, and * denotes significant difference between means. 

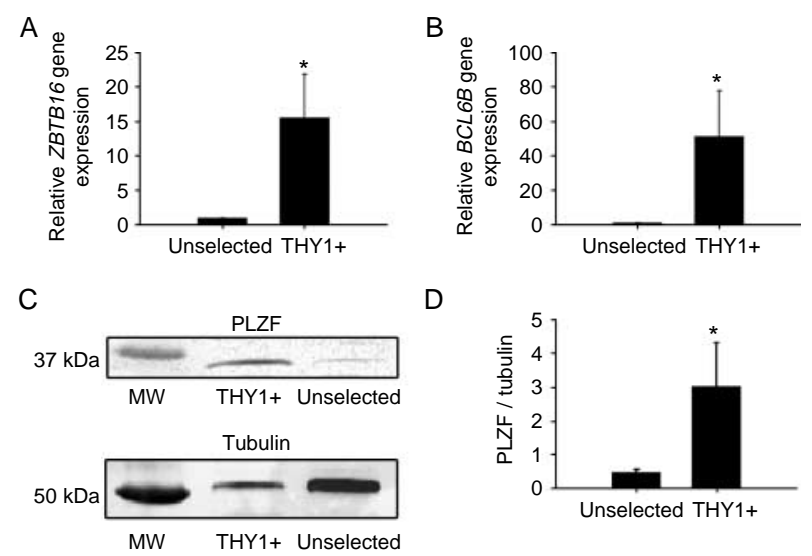

D

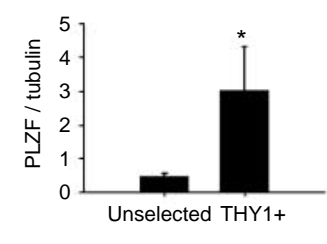

Figure 5 Examination of the MACS-isolated THY1 + cell fraction from pre-pubertal bull testes for expression of genes restricted to the undifferentiated spermatogonial population. (A) Relative ZBTB16 (PLZF) gene expression in MACS-isolated THY1 + cell fractions compared to unselected total testis cell populations measured by qRT-PCR. Data are mean \pm s.E.M. for three different cell preparations, and ${ }^{*}$ denotes significant difference between means. ZBTB16 expression was $15.6 \pm 6.3$-fold greater in MACS-isolated THY1 + cell fractions compared to unselected total testis cell populations. Data are mean \pm S.E.M. for three different cell preparations. (B) Relative BCL6B gene expression in MACS-isolated THY1 + cell fractions compared to unselected total testis cell population measured by qRT-PCR. Data are mean \pm S.E.M. for three different cell preparations, and $*$ denotes significance between means. Expression was 51.3 \pm 26.8 -fold greater in MACS-isolated THY1 + cell fractions compared to unselected total testis cell populations. Data are mean \pm s.E.M. for three different cell preparations. Comparison between means for both ZBTB16 and BCL6B gene expressions was made by normalizing to expression of the constitutively active gene RPS2. (C) Representative images of western blot analysis for PLZF protein expression in MACS-isolated $\mathrm{THY} 1+$ fractions and unselected total testis cell populations. A distinct band of $\sim 36 \mathrm{kDa}$ was identified for the bovine testis. (D) Quantitative comparison of western blot analyses for PLZF protein expression. Data are mean \pm S.E.M. for three different cell preparations, and ${ }^{*}$ denotes significant difference between means. Comparison between means was made by normalizing PLZF band density in each sample to that of $\beta$-tubulin. PLZF protein expression was determined to be $8.2 \pm 5.0$-fold greater in MACS-isolated THY1 + cells compared to unselected total testis cell populations.

in testes of recipient mice (Fig. 6). Colonies consisting of cohorts of greater than four cells extending along the periphery of seminiferous tubules were readily identified for transplanted THY1 + cells indicating migration from the lumen to the basement membrane and proliferation (Fig. 6A and B). Colonies generated from unselected cells were generally smaller consisting of groups of cells along the periphery of tubules (Fig. 6C and D). Quantification of the colonies revealed that THY1+ cells generated a significantly $(P=0.009)$ greater number of colonies compared to the unselected total testis cell population (Fig. 6E). Overall, the THY1 + cells generated greater than sixfold $\left(94.0 / 14.4\right.$ colonies $/ 10^{5}$ cells transplanted; $n=3$ replicate experiments) more colonies than the unselected total testis cell population. These results demonstrate that the MACS-isolated THY1 + cell fraction from pre-pubertal bull testes is enriched for cells capable of colonization following transplantation into recipient seminiferous tubules compared to the unselected total testis cell population suggesting enrichment for SSC content (Fig. 6).

\section{Discussion}

Defining spermatogonial populations expands the knowledge of male fertility and aids in developing technologies to enhance reproductive efficiency. Use of markers to study the rare SSCs and other undifferentiated spermatogonial sub-types is a critical tool for making novel discoveries. Previous studies in rodents and nonhuman primates showed that PLZF is a molecular marker of the undifferentiated spermatogonial population that includes SSCs (Buaas et al. 2004, Costoya et al. 2004), but expression of this molecule has yet to be explored in bulls. UCHL1, also referred to as PGP9.5, is an established marker of porcine and bovine type A spermatogonia (Herrid et al. 2007). In this study, we investigated PLZF expression in relation to UCHL1-expressing cells to determine whether PLZF could serve as a marker of undifferentiated spermatogonia in bulls. Consistent with the pattern of expression previously observed for mice (Buaas et al. 2004, Costoya et al. 2004, Payne \& Braun 2006, Takubo et al. 2008, Luo et al. 2009), PLZF expression was observed in the nucleus of spermatogonia but was also seen in the cytoplasm of some spermatogonia. This heterogeneity of PLZF localization may be a unique expression pattern in the bull and other livestock and could be reflective of spermatogonia in different stages of the development or proliferation. Quantitative analyses indicate that PLZF-expressing cells are a sub-population of type A spermatogonia within the more abundant UCHL1+ population of pre-pubertal bull testes. This finding suggests that UCHL1 is expressed by a majority of type A spermatogonia in pre-pubertal bulls, whereas PLZF is expressed by a sub-population of spermatogonia and could be more restricted to SSCs. Thus, similar to rodents, PLZF is an effective marker for studying the undifferentiated spermatogonial population in bulls.

Results of the present study demonstrate that selection of THY1 + cells yields a cell fraction enriched for undifferentiated spermatogonia from testes of prepubertal bulls. The MACS-isolated THY1 + cell fraction was found to be composed mostly ( $>60 \%$ ) of PLZF + spermatogonia. Surprisingly, the unselected total testis cell population in pre-pubertal bulls contained a higher than expected percentage of PLZF + cells (i.e. $\sim 20 \%$ ), which was likely due to processing of the samples because initial collagenase digestion was conducted to reduce interstitial cells and enrich for cells within seminiferous tubules. Regardless, selection of THY1+ cells resulted in nearly threefold enrichment of PLZF+ cells. In accordance with greater PLZF + germ cell 
A

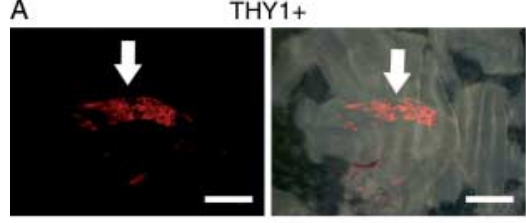

C

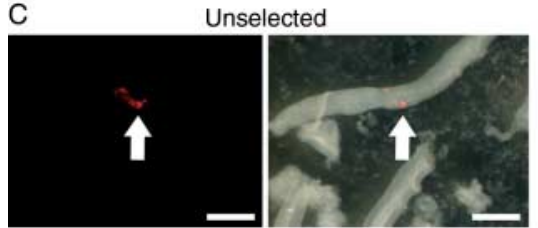

E

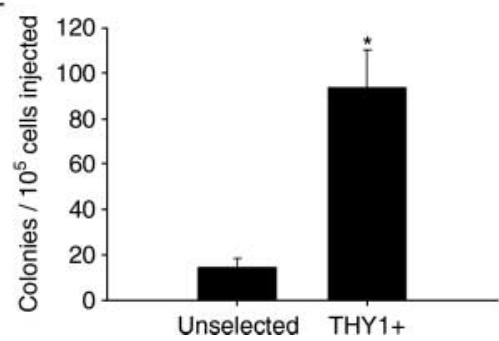

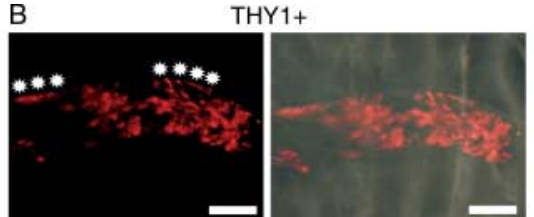

D Unselected

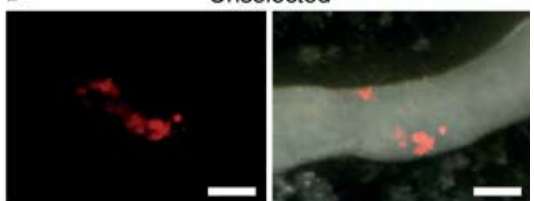

Figure 6 Examination of the MACS-isolated THY1 + cell fraction from pre-pubertal bull testes for colonizing ability in recipient testes following xenogeneic transplantation. MACS-isolated THY1 + and unselected total testis cell populations were labeled with PKH26 red fluorescent dye and microinjected into the seminiferous tubules of recipient immunodeficient nude mice. (A-D) Representative images of bovine germ cell colonies (arrows) within recipient mouse seminiferous tubules transplanted with MACS-isolated THY1+ cells (A and B) or unselected total testis cell populations ( $C$ and D). For each panel, the left image is fluorescent view, and right image is overlay of fluorescent view with the corresponding bright field image. Image B is a magnification of A and D is a magnification of C. Colonies consisting of groups or chains of cells (stars) at the periphery of seminiferous tubules were seen indicating migration to the basement membrane and proliferation. Bars are $2 \mathrm{~mm}$. (E) Quantitative comparison of bovine germ cell colonies in recipient mouse testes generated by transplantation of MACS-isolated THY1 + or unselected total testis cell populations. Data are mean \pm s.E.M. for three independent experiments, and * denotes significant difference between means.

content, xenogeneic transplantation assays showed that the THY $1+$ fractions are enriched for germ cells capable of colonization which is indicative of SSC activity. Collectively, these results demonstrate that THY1 is a conserved surface marker of undifferentiated spermatogonia and likely SSCs in the bull. Importantly, selection of THY $1+$ cells from testes of rodents and non-human primates also results in enrichment of these cells (Kubota et al. 2003, Ryu et al. 2004, Hermann et al. 2009). Moreover, expression of BCL6B is enriched in the $\mathrm{THY} 1+$ germ cell fraction from testes of both bulls and mice. Thus, expression of THY1 is a common phenotype of SSCs in several mammalian species and it is also likely conserved in other livestock.

In addition to THY1, other cell surface markers of undifferentiated spermatogonia have been identified in rodents including a6-integrin (Shinohara et al. 1999, 2000), GFRA1 (GFR $\alpha 1$; Meng et al. 2000, Buageaw et al. 2005, Ebata et al. 2005, Hofmann et al. 2005, Gassei et al. 2009), and CD9 (Kanatsu-Shinohara et al. 2004). Transplantation analyses showed that in adult mouse testes, the $\alpha 6$-integrin + and CD9+ cell fractions are enriched approximately sevenfold and eightfold for SSCs respectively (Shinohara et al. 1999, KanatsuShinohara et al. 2004). In pre-pubertal mouse pups, the
GFRA $1+$ cell fraction is enriched approximately twofold for SSCs (Buageaw et al. 2005, Ebata et al. 2005); however, this phenotype is not retained in adult mouse testes in which the GFRA1 + cell population is actually depleted of SSCs (Ebata et al. 2005, Grisanti et al. 2009). SSC content of the $\alpha 6$-integrin- and CD9-expressing cell fractions of pre-pubertal mouse pups has not been reported. In comparison, the THY1+ cell fraction is enriched 5- and 30-fold in pre-pubertal and adult mouse testes respectively (Kubota et al. 2004). Thus, of the identified cell surface markers of the heterogeneous undifferentiated spermatogonial population in rodent testes, functional transplantation studies have shown THY1 to be the most specific marker of SSCs (Oatley \& Brinster 2008). Expression of $\alpha 6$-integrin, GFRA1, and CD9 in the bull testis has not been reported, but results of the current study show that THY1 is a conserved marker of undifferentiated spermatogonia in the pre-pubertal bull testis.

Development of reproductive tools utilizing SSCs (e.g. long-term culture and transplantation) relies on isolation of these rare cells. Use of THY1 as a marker for bovine SSCs will aid in progressing the development of these tools in cattle. Maintaining bovine SSCs in culture could provide a means for immortalizing the germline of 
genetically superior sires. Transplantation of SSCs has the potential to provide an alternative-assisted reproductive technique in the beef cattle industry, where the use of artificial insemination (AI) has been limited due to impracticality. Isolation of SSCs from a genetically desirable donor bull followed by transplantation into a battery of recipient bulls that subsequently produce donor sperm and are used for natural breeding could provide a novel means to expand the utilization of specific genetics within commercial cattle populations. Both of these tools have the potential to increase efficiency of cattle production; thus, decreasing wastes, costs, and environmental impacts of producing food and fiber for human consumption. Additionally, these tools could provide a novel avenue for genetic modification of the male germline and subsequent generation of transgenic livestock with favorable traits such as disease resistance and production of meat or milk containing components beneficial for human consumption. The current study provides a crucial first step in achieving these goals by establishing a means for isolating a cell fraction that is enriched for undifferentiated spermatogonia from bull testes.

The advancement of reproductive tools based on SSCs is at the forefront of development in livestock. In rodents, progression of techniques for isolation, culture, and transplantation of SSCs spanned greater than a decade of research. The first successful transplantation of SSCs was reported by pioneering work of Brinster \& Avarbock (1994) and Brinster \& Zimmermann (1994). Cryopreservation of mouse SSCs was first reported 2 years later by Avarbock et al. (1996), and initial evidence that mouse SSCs could be maintained for long periods of time in vitro was reported 2 years after that by Nagano et al. (1998). Shinohara et al. (1999) reported the first cell surface markers of mouse SSCs. Nagano et al. (2001) reported the production of transgenic mice after transplantation of genetically modified SSCs into testes of infertile recipients. Two years later, Kubota et al. (2003) discovered that the cell surface marker THY1 could be used to isolate an enriched population of SSCs from mouse testes. Presently, THY1 isolation continues to yield the greatest enrichment of SSCs from the mouse testis (Oatley \& Brinster 2008). Also, Kanatsu-Shinohara et al. (2003) reported the long-term culture of gonocytes from mouse testes. Kubota et al. (2004) reported conditions for long-term maintenance of mouse SSCs in vitro, with similar methodology being identified for rats 1 year later (Ryu et al. 2005). Here, we describe a means to isolate an enriched population of undifferentiated spermatogonia from the pre-pubertal bull testis based on THY1 expression. This achievement is a crucial advancement for furthering the development of SSC applications in bulls. Based on the timeline of developments in rodents, major advances in culturing and transplanting bovine SSCs should be made in the coming decade.

\section{Materials and Methods}

\section{Magnetic activated cell sorting of THY1 + testis cells}

All animal procedures were approved by the Pennsylvania State University Institutional Animal Care and Use Committee. Single cell suspensions of testis cells were collected from 12- to 14-week-old Holstein bull calves by two-step enzymatic digestion. At these ages, gonocytes and undifferentiated spermatogonia are the only germ cell types present and can be referred to as the primitive spermatogonial population. Thus, the testis cell population at this age is optimal for studying characteristics of SSCs. Approximately, 200-300 mg of testis tissue was incubated in collagenase solution $(1 \mathrm{mg} / \mathrm{ml}$ collagenase type 4; Worthington Biochemical Corporation; Lakewood, NJ, USA and $7 \mathrm{mg} / \mathrm{ml}$ DNase I, Sigma Inc.) at $37^{\circ} \mathrm{C}$ for $30 \mathrm{~min}$ with agitation every $2 \mathrm{~min}$. Seminiferous tubule fragments were then allowed to settle on ice and washed $3 \times$ with Hank's balanced salt solution to remove interstitial cells. Single cell suspensions of tubules were then created by incubation in trypsin-EDTA solution $(0.25 \%$ trypsin/2.21 mM EDTA; Cellgro Mediatech Inc., Manassas, VA, USA) containing DNase I ( $7 \mathrm{mg} / \mathrm{ml})$ for $5 \mathrm{~min}$ at $37^{\circ} \mathrm{C}$. Cells were then passed through a $40 \mu \mathrm{m}$ cell strainer (BD Biosciences, Durham, NC, USA) to remove clumps and pelletted by centrifugation at $600 \mathrm{~g}$ for $7 \mathrm{~min}$. Cells were then resuspended in DPBS-S (PBS with $1 \%$ FBS, $10 \mathrm{mM}$ HEPES, $1 \times 10^{4} \mathrm{U} / \mathrm{ml}$ penicillin, $1 \times 10^{4} \mu \mathrm{g} / \mathrm{ml}$ streptomycin, $1 \mathrm{mM}$ sodium pyruvate, and $1 \mathrm{mg} / \mathrm{ml}$ glucose), and cell concentration was determined using a hemocytometer with trypan blue exclusion to identify live cells. Aliquots of $1 \times 10^{7}$ cells were incubated with mouse antihuman THY1 antibody (1:10; Abcam, Cambridge, MA, USA) for $20 \mathrm{~min}$ at $4{ }^{\circ} \mathrm{C}$. Cells were then washed $2 \times$ with DPBS-S by centrifugation at $600 \mathrm{~g}$ for $7 \mathrm{~min}$, followed by incubation with biotinylated goat anti-mouse antibody $(1: 100$; Santa Cruz Biotechnology Inc., Santa Cruz, CA, USA) at $4{ }^{\circ} \mathrm{C}$ for $20 \mathrm{~min}$. Cells were again washed $2 X$ in DPBS-S with centrifugation and then incubated with strepavidin-conjugated antibody to magnetic microbeads (1:10; Miltenyi Biotec, Auburn, CA, USA) for $20 \mathrm{~min}$ on ice. Cells were subjected to another two washes in DPBS-S using centrifugation, and THY1 + cells were collected by MACS (Miltenyi Biotec). Typical yields of $2-4 \times 10^{5}$ THY $1+$ cells were isolated from 200 to $300 \mathrm{mg}$ of testis tissue. For comparison, single cell suspensions not subjected to THY1 MACS isolation (referred to as unselected total testis cell populations) were collected from identical tissue used for THY1 + cell isolation.

\section{Flow cytometric analyses of THY1-expressing cells}

The percentage of cells in pre-pubertal bull testes that express THY1 was evaluated using FCA. Single cell suspensions of bovine testicular tissue were collected as described above and incubated with mouse anti-human THY1 (1:100; Abcam) primary antibody. Secondary detection involved incubation with Alexa 488-conjugated donkey anti-mouse IgG (1:1000; R\&D Systems, Emeryville, CA, USA) antibody. The percentage of THY1-expressing cells was determined using a Guava EasyCyte Plus flow cytometer (Millipore Corporation, Billerica, 
MA, USA). Cell suspensions from identical samples analyzed for THY1 not incubated with primary antibody served as negative controls.

\section{Immunohistochemical analysis of THY1-expressing cells within seminiferous tubules of pre-pubertal bulls}

Testis samples were collected from 12- to 14-week-old Holstein bull calves, fixed in Bouin's solution, dehydrated, and embedded in paraffin, and $5 \mu \mathrm{m}$ cross sections were adhered to glass slides. After deparaffinization and rehydration, sections were blocked for endogenous biotin with a biotinblocking kit (Invitrogen). Sections were then blocked for non-specific antibody binding by incubation with $10 \%$ normal goat serum in PBS for $1 \mathrm{~h}$ followed by incubation with mouse anti-human THY1 (1:50; Abcam) primary antibody at $4{ }^{\circ} \mathrm{C}$ overnight. Negative controls were cross sections incubated with normal mouse IgG (1:50; Santa Cruz Biotechnology) in place of primary antibody. On the next day, sections were washed $3 \times$ in PBS followed by incubation with biotinylated goat anti-mouse IgG (1:200; Santa Cruz Biotechnology) secondary antibody for $2 \mathrm{~h}$ at room temperature. Sections were again washed $3 X$ in PBS and incubated with an avidin-HRP conjugate (Vectastain Universal Elite ABC Kit; Vector Laboratories, Burlingame, CA, USA) for $10 \mathrm{~min}$ and washed $3 \times$ in PBS. Cross sections were then incubated in a $3,3^{\prime}$ diaminobenzidine (DAB) substrate to develop peroxidase activity (DAB Substrate Kit; Vector Laboratories) followed by washing $3 \times$ with distilled water and counterstaining with hematoxylin. Sections were then washed with tap water and mounted with glycerol vinyl alcohol (GVA) compound (Invitrogen). All samples were viewed using bright field microscopy, and digital images were captured with an Olympus DP71 microscope camera (Olympus America Inc., Center Valley, PA, USA).

\section{Immunofluorescence analyses of PLZF- and UCHL1-expressing cells}

Expression of PLZF and UCHL1 was localized in cross sections of pre-pubertal bull testes using immunofluorescence analyses. Testis samples were collected from 12- to 14-weekold Holstein bull calves, fixed in Bouin's solution, dehydrated, and embedded in paraffin, and $5 \mu \mathrm{m}$ cross sections were adhered to glass slides. After deparaffinization and rehydration, antigen retrieval was achieved by boiling in citrate buffer (10 mM citric acid and $0.05 \%$ Tween-20) for $20 \mathrm{~min}$. Sections were then blocked for non-specific antibody binding by incubation with $10 \%$ normal goat serum in PBS for $1 \mathrm{~h}$. Tissues were then incubated with goat anti-human PLZF (1:50; Santa Cruz Biotechnology) and/or rabbit anti-human UCHL1 (1:500; Abcam) primary antibodies at $4{ }^{\circ} \mathrm{C}$ overnight. Controls were cross sections incubated with normal goat IgG (1:50; Santa Cruz Biotechnology) or normal rabbit IgG (1:500; Santa Cruz Biotechnology) in place of primary antibodies at $4{ }^{\circ} \mathrm{C}$ overnight. On the next day, sections were washed $3 \times$ in PBS followed by incubation with Alexa 488-conjugated donkey anti-goat IgG (1:1000; Invitrogen Inc.) or Alexa 546-conjugated donkey anti-rabbit IgG (1:1000; Invitrogen Inc.) for $2 \mathrm{~h}$ at room temperature. Sections were again washed $3 \times$ in PBS and counterstained with DAPI to label cell nuclei. Sections were then examined using fluorescent microscopy (Axioskop 2 Plus, Zeiss Inc., Thornwood, NY, USA), and digital images were captured with an Olympus DP71 microscope camera (Olympus America Inc). For each replicate testis sample $(n=3$ different bulls), the number of PLZF + and/or UCHL1 + cells within each round seminiferous tubule of five random fields of view was counted. The total number of round tubules within each field was also counted to determine the average number of PLZF + and/or UCHL1 + cells per seminiferous tubule. In total, 40 round tubules were examined from three replicate samples.

\section{Quantitative RT-PCR analyses of ZBTB16 (PLZF) and BCL6B gene expression}

Expression of specific genes of interest by MACS-isolated THY1 + testis cells and unselected total testis cell populations was examined using qRT-PCR analyses. RNA was isolated using TRIzol reagent (Sigma Inc.) followed by treatment with DNase I (DNA-free kit; Ambion, Austin, TX, USA) to remove possible contaminating genomic DNA. RNA concentration in each sample was measured by spectrophotometry (Nano Drop1000, Nano Drop Technologies Inc., Wilmington, DE, USA), and 260/280 ratios were calculated to determine RNA purity. For each sample, $500 \mathrm{ng}$ of RNA was reverse transcribed using oligo $d(T)$ priming and M-MLV RT (Superscript III first strand synthesis kit; Invitrogen Inc). Quality of resulting cDNAs was determined by conventional PCR analyses for expression of glyceraldhehyde 3-phosphate dehydrogenase $(G A P D H)$. Primers for ZBTB16, B-cell CLL/lymphoma 6, member B $(B C L 6 B)$, and ribosomal protein S2 (RPS2) were designed using Primer Express 3 software (Applied Biosystems, Foster City, CA, USA) and are reported in Table 1. Relative expression levels of ZBTB16 and BCL6B were then determined by qRT-PCR analyses with SYBR green assays (Invitrogen Inc.) and a 7500 Fast Sequence Detection System (Applied Biosystems). To make comparisons between MACS-isolated $\mathrm{THY} 1+$ and unselected total testis cell populations, the level of expression for each gene of interest in each sample was normalized to that of the constitutively active gene RPS2 as described previously (Oatley et al. 2006, 2007).

Table 1 Primer sequences for quantitative reverse transcription-PCR analyses.

\begin{tabular}{lll}
\hline Gene & Forward primer $\left(5^{\prime}-3^{\prime}\right)$ & Reverse primer $\left(5^{\prime}-3^{\prime}\right)$ \\
\hline BCL6B & AGGGCACAGGGAACTCTTTTC & CCTCCTTTGGCTTGAGTGTTTT \\
ZBTB16 $(P L Z F)$ & CCAGCAGATTCTGGAGTATGCA & GCATACAGCAGGTCATCCAAGTC \\
RPS2 & GGAGCATCCCTGAAGGATGA & TCCCCGATAGCAACAAACG \\
\hline
\end{tabular}




\section{Western blot analyses of PLZF protein expression}

Expression of PLZF protein in MACS-isolated THY1 + testis cells and unselected total testis cell populations was examined using western blot analyses. Protein lysates were collected using mammalian protein extraction reagent (Thermo Scientific, Logan, UT, USA). For each sample, $\sim 30-50 \mu$ g of protein was separated by SDS-PAGE and transferred to nitrocellulose membranes. Blots were blocked with $5 \%$ non-fat dry milk powder in PBS containing 0.05\% Tween-20 for $2 \mathrm{~h}$. Blots were then incubated with rabbit anti-human PLZF antibody (1:2000; Santa Cruz Biotechnology Inc.) in PBS with $0.05 \%$ BSA and $0.1 \%$ Triton $\mathrm{X}-100$ overnight at $4{ }^{\circ} \mathrm{C}$. The next day, blots were washed $3 \times$ with Tris-buffered saline containing $0.1 \%$ Tween20 (TBS-T) followed by incubation with goat anti-rabbit IgG conjugated to HRP (1:2500; Santa Cruz Biotechnology Inc). Blots were again washed $3 \times$ with TBS-T, developed with SuperSignal West Pico Chemiluminescent Substrate (Pierce, Rockford, IL, USA), viewed using a ChemiDoc imager (Universal Hood II, Bio-Rad Laboratories Inc.), and digital images were captured. To make quantitative comparisons between MACS-isolated THY1 + testis cells and unselected total testis cell populations, blots were re-probed with a rabbit anti-human $\beta$-tubulin primary antibody (1:5000; Novus Biologicals, Inc., Littleton, CO, USA). Secondary detection included incubation with HRP-conjugated goat anti-rabbit IgG and developed with chemiluminescent substrate. To make quantitative comparisons between samples, PLZF band densities were normalized to corresponding densities of $\beta$-tubulin using Quantity One analysis software (Bio-Rad Inc).

\section{Immunocytochemical analyses for PLZF- and VASA-expressing testis cells}

Immunocytochemical staining was conducted to determine the percentage of PLZF + and VASA + spermatogonia in MACSisolated THY1 + testis cell fractions and unselected total testis cell populations. Isolated THY1 + and unselected total testis cells were adhered to glass cover slips followed by fixation in $4 \%$ paraformaldehyde for $10 \mathrm{~min}$ at room temperature. Non-specific antibody binding was blocked by incubating cells in $10 \%$ normal donkey or normal goat serum for $1 \mathrm{~h}$ at room temperature. Cells were then incubated with goat antihuman PLZF antibody (1:100; Santa Cruz Biotechnology Inc.) or rabbit anti-human VASA (1:100; Abcam) in PBS with $0.05 \%$ BSA and $0.1 \%$ Triton $X-100$ at $4{ }^{\circ} \mathrm{C}$ overnight. On the next day, cells were washed $3 \times$ with PBS and incubated with Alexa 488-conjugated donkey anti-goat or goat anti-rabbit IgG (1:1000; Invitrogen Inc.) for $2 \mathrm{~h}$. Cells were again washed $3 \times$ with PBS and incubated with DAPI for 3 min to stain cell nuclei. Cover slips were then visualized by fluorescent microscopy at $20 \times$ magnification. For each replicate sample, the total number of DAPI-stained nuclei in five random fields of view was counted. The number of PLZF + and VASA + cells was also counted in each field, and the average percentage of $\mathrm{PLZF}+$ and VASA + cells were determined by dividing the number of DAPI-stained nuclei in each field by the number of PLZF + or VASA + cells. For each analysis, $>150$ cells were evaluated.

\section{Xenogeneic transplantation of bull testis cells and analysis of colonization}

Xenogeneic transplantation assays were conducted to compare SSC content of MACS-isolated THY1+ cells to unselected total testis cell populations of pre-pubertal bull testes as described previously (Oatley et al. 2004a, 2004b). Briefly, recipient NCr Swiss nude mice (Taconic, Germantown, NY, USA) were treated with busulfan $(33 \mathrm{mg} / \mathrm{kg}$ of body weight) 6 weeks prior to transplantation to deplete endogenous spermatogenesis. Following enzymatic digestion and MACS isolation, THY1 + and unselected total testis cell populations were resuspended in PBS and labeled with PKH26 red fluorescent cell linker dye following the manufacturer's instructions (Sigma Inc). PKH26 stably incorporates as a fluorescent molecule into lipid regions of the cell membrane and is retained for several rounds of proliferation. Cells were then washed $5 \times$ in DPBS-S by centrifugation at $600 \mathrm{~g}$ for $7 \mathrm{~min}$ to remove residual dye. For each sample, cells were resuspended in DPBS-S at a concentration of $3 \times 10^{6}$ cells $/ \mathrm{ml}$, and $3-7 \mu \mathrm{l}$ of cell suspension (i.e. $3-7 \times 10^{4}$ cells) was microinjected into the seminiferous tubules of each recipient testis. Three recipient mice (i.e. six testes) were transplanted with each replicate cell suspension, and three replicate experiments were performed totaling 9 mice and 18 testes that were transplanted with MACS-isolated THY1+ testis cells or unselected total testis cell populations. Recipient mice were euthanized 4 weeks after transplantation, and testes were recovered and incubated with collagenase (type 4, $1 \mathrm{mg} / \mathrm{ml})$ and DNase I $(7 \mathrm{mg} / \mathrm{ml})$ to separate seminiferous tubules followed by washing $3 \times$ in DPBS-S. Seminiferous tubules were then suspended in a small volume of DPBS-S, spread on $10 \mathrm{~cm}$ dishes, and analyzed for colonization of bovine testicular cells using a fluorescent stereomicroscope equipped with a TRITC filter set (Olympus SZX10). The number of fluorescently labeled colonies of bovine cells was counted for each recipient testis, and digital images were captured with a DP71 microscope imaging system. To make quantitative comparisons between MACS-isolated THY1+ testis cells and unselected total testis cell populations, the number of colonies generated by each sample was normalized to $1 \times 10^{5}$ cells microinjected into recipient mouse testes.

\section{Statistical analyses}

Data were analyzed using the General Linear Model or mixed model function of SPSS statistical software (Chicago, IL, USA). Differences between means were determined using a univariate ANOVA in conjunction with Tukey-Kramer multiple comparisons test. Data were considered significantly different at $P \leq 0.05$.

\section{Declaration of interest}

The authors declare that there is no conflict of interest that could be perceived as prejudicing the impartiality of the research reported. 


\section{Funding}

This work was supported by the United States Department of Agriculture (grant number 2008-35203-19073).

\section{Acknowledgements}

We would like to thank Amy Kaucher for helpful suggestions to improve the manuscript.

\section{References}

Aponte PM, Soda T, Teerds KJ, Mizrak SC, van de Kant HJ \& de Rooij DG 2008 Propagation of bovine spermatogonial stem cells in vitro. Reproduction 136 543-557.

Avarbock MR, Brinster CL \& Brinster RL 1996 Reconstitution of spermatogenesis from frozen spermatogonial stem cells. Nature Medicine 2 638-639.

Bartholomew RA \& Parks JE 2007 Identification, localization, and sequencing of fetal bovine vasa homolog. Animal Reproduction Science 101 241-251.

Brinster RL \& Avarback MR 1994 Germline transmission of donor haplotype following spermatogonial transplantation. PNAS 91 11303-11307.

Brinster RL \& Zimmermann JW 1994 Spermatogenesis following male germ-cell transplantation. PNAS 91 11298-11302.

Buaas FW, Kirsh AL, Sharma M, McLean DJ, Morris JL, Griswold MD, de Rooij DG \& Braun RE 2004 Plzf is required in adult male germ cells for stem cell self-renewal. Nature Genetics 36 647-652.

Buageaw A, Sukhwani M, Ben-Yehudah A, Ehmcke J, Rawe VY, Pholpramool C, Orwig KE \& Schlatt S 2005 GDNF family receptor alpha1 phenotype of spermatogonial stem cells in immature mouse testes. Biology of Reproduction 73 1011-1016.

Costoya JA, Hobbs RM, Barna M, Cattoretti G, Manova K, Sukhwani M, Orwig KE, Wolgemuth DJ \& Pandolfi PP 2004 Essential role of Plzf in maintenance of spermatogonial stem cells. Nature Genetics 36 551-553.

Curtis SK \& Amann RP 1981 Testicular development and establishment of spermatogenesis in holstein bulls. Journal of Animal Science $\mathbf{5 3}$ 1645-1657.

Dobrinski I, Avarbock MR \& Brinster RL 2000 Germ cell transplantation from large domestic animals into mouse testes. Molecular Reproduction and Development 57 270-279.

Ebata KT, Zhang X \& Nagano MC 2005 Expression patterns of cell-surface molecules on male germ line stem cells during postnatal mouse development. Molecular Reproduction and Development 72 171-181.

Frankenhuis MT, Kramer MF \& de Rooij DG 1982 Spermatogenesis in the boar. Veterinary Quarterly 4 57-61.

Gassei K, Ehmcke J \& Schlatt S 2009 Efficient enrichment of undifferentiated GFR alpha $1+$ spermatogonia from immature rat testis by magnetic activated cell sorting. Cell Tissue Research 337 177-183.

Grisanti L, Falciatori I, Grasso M, Dovere L, Fera S, Muciaccia B, Fuso A, Berno V, Boitani C, Stefanini M et al. 2009 Identification of spermatogonial stem cell subsets by morphological analysis and prospective isolation. Stem Cells 27 3043-3052.

Hermann BP, Sukhwani M, Lin CC, Sheng Y, Tomko J, Rodriguez M, Shuttleworth JJ, McFarland D, Hobbs RM, Pandolfi PP et al. 2007 Characterization, cryopreservation, and ablation of spermatogonial stem cells in adult rhesus macaques. Stem Cells 25 2330-2338.

Hermann BP, Sukhwani M, Simorangkir DR, Chu T, Plant TM \& Orwig KE 2009 Molecular dissection of the male germ cell lineage identifies putative spermatogonial stem cells in rhesus macaques. Human Reproduction 24 1704-1716.

Herrid M, Davey RJ \& Hill JR 2007 Characterization of germ cells from pre-pubertal bull calves in preparation for germ cell transplantation. Cell Tissue Research 330 321-329.

Hofmann MC, Braydich-Stolle L \& Dym M 2005 Isolation of male germline stem cells; influence of GDNF. Developmental Biology 279 114-124.
Huckins C 1971 The spermatogonial stem cell population in adult rats. I. Their morphology, proliferation and maturation. Anatomical Record 169 533-557.

Izadyar F, Den Ouden K, Stout TA, Stout J, Coret J, Lankveld DP, Spoormakers TJ, Colenbrander B, Oldenbroek JK, Van der Ploeg KD et al. 2003 Autologous and homologous transplantation of bovine spermatogonial stem cells. Reproduction 126 765-774.

Kanatsu-Shinohara M, Ogonuki N, Inoue K, Miki H, Ogura A, Toyokuni S \& Shinohara T 2003 Long-term proliferation in culture and germline transmission of mouse male germline stem cells. Biology of Reproduction 69 612-616.

Kanatsu-Shinohara M, Toyokuni S \& Shinohara T 2004 CD9 is a surface marker on mouse and rat male germline stem cells. Biology of Reproduction 70 70-75.

Kubota H, Avarbock MR \& Brinster RL 2003 Spermatogonial stem cells share some, but not all, phenotypic and functional characteristics with other stem cells. PNAS 100 6487-6492.

Kubota H, Avarbock MR \& Brinster RL 2004 Culture conditions and single growth factors affect fate determination of mouse spermatogonial stem cells. Biology of Reproduction 71 722-731.

Lee GS, Kim HS, Lee SH, Kang MS, Kim DY, Lee CK, Kang SK, Lee BC \& Hwang WS 2005 Characterization of pig vasa homolog gene and specific expression in germ cell lineage. Molecular Reproduction and Development 72 320-328.

Luo J, Megee S, Rathi R \& Dobrinski I 2006 Protein gene product 9.5 is a spermatogonia-specific marker in the pig testis: application to enrichment and culture of porcine spermatogonia. Molecular Reproduction and Development 73 1531-1540.

Luo J, Megee S \& Dobrinski I 2009 Asymmetric distribution of UCH-L1 in spermatogonia is associated with maintenance and differentiation of spermatogonial stem cells. Journal of Cellular Physiology 220 460-468.

Meng X, Lindahl M, Hyvönen ME, Parvinen M, de Rooij DG, Hess MW, Raatikainen-Ahokas A, Sainio K, Rauvala H, Lakso M et al. 2000 Regulation of cell fate decision of undifferentiated spermatogonia by GDNF. Science 287 1489-1493.

Nagano M, Avarbock MR, Leonida EB, Brinster CJ \& Brinster RL 1998 Culture of mouse spermatogonial stem cells. Tissue Cell 30 389-397.

Nagano M, Brinster CJ, Orwig KE, Ryu BY, Avarbock MR \& Brinster RL 2001 Transgenic mice produced by retroviral transduction of malegermline stem cells. PNAS 98 13090-13095.

Oakberg EF 1971 Spermatogonial stem cell renewal in the mouse. Anatomical Record 169 515-531.

Oatley JM \& Brinster RL 2008 Regulation of spermatogonial stem cells selfrenewal in mammals. Annual Review of Cell and Developmental Biology 24 263-286.

Oatley JM, de Avila DM, McLean DJ, Griswold MD \& Reeves JJ 2002 Transplantation of bovine germinal cells into mouse testes. Journal of Animal Science 80 1925-1931.

Oatley JM, Reeves JJ \& McLean DJ 2004a Biological activity of cryopreserved bovine spermatogonial stem cells during in vitro culture. Biology of Reproduction 71 942-947.

Oatley JM, de Avila DM, Reeves JJ \& McLean DJ 2004b Testis tissue explant culture supports survival and proliferation of bovine spermatogonial stem cells. Biology of Reproduction 70 625-631.

Oatley JM, Avarbock MR, Telaranta AI, Fearon DT \& Brinster RL 2006 Identifying genes important for spermatogonial stem cell self-renewal and survival. PNAS 103 9524-9529.

Oatley JM, Avarbock MR \& Brinster RL 2007 Glial cell line-derived neurotrophic factor regulation of genes essential for self-renewal of mouse spermatogonial stem cells is dependent on SRC family kinase signaling. Journal of Biological Chemistry 282 25842-25851.

Payne C \& Braun RE 2006 Histone lysine trimethylation exhibits a distinct perinuclear distribution in Plzf-expressing spermatogonia. Developmental Biology 293 461-472.

Ryu BY, Orwig KE, Kubota H, Avarbock MR \& Brinster RL 2004 Phenotypic and functional characteristics of spermatogonial stem cells in rats. Developmental Biology 274 158-170.

Ryu BY, Kubota H, Avarbock MR \& Brinster RL 2005 Conservation of spermatogonial stem cell self-renewal signaling between mouse and rat. PNAS 102 14302-14307. 
Sharpe R 1994 Regulation of spermatogenesis. In The Physiology of Reproduction, edn 2, pp 1363-1434. Eds E Knobil \& JD Neill. New York: Raven Press.

Shinohara T, Avarbock MR \& Brinster RL 1999 ß1- and a6-intergrin are surface markers and mouse spermatogonial stem cells. PNAS 96 5505-5509.

Shinohara T, Orwig KE, Avarbock MR \& Brinster RL 2000 Spermatogonial stem cell enrichment by multiparameter selection of mouse testis cells. PNAS 97 8346-8351.

Takubo K, Ohmura M, Azuma M, Nagamatsu G, Yamada W, Arai F, Hirao A \& Suda T 2008 Stem cell defects in ATM-deficient undifferentiated spermatogonia through DNA damage-induced cell-cycle arrest. Cell Stem Cell 2 170-182.
Toyooka Y, Tsuenekawa N, Takahashi Y, Matsui Y, Satoh M \& Noce T 2000 Expression and intercellular localization of mouse vasa-homologue protein during germ cell development. Mechanisms of Development 93 139-149.

Received 17 November 2009

First decision 2 December 2009

Revised manuscript received 23 January 2010

Accepted 12 February 2010 